Digital Object Identifier (DOI): 10.38087/2595.8801.50

\title{
EPIDEMIOLOGIA: CORONAVÍRUS (COVID-19) E RECOMENDAÇÕES DA PRÁTICA DE ATIVIDADE FÍSICA E EXERCÍCIO FÍSICO.
}

Cassio Hartmann ${ }^{1}$

Gabriel César Dias Lopes²

Fábio da Silva Ferreira Vieira ${ }^{3}$

Bensson V Samuel ${ }^{4}$

\section{RESUMO}

O novo Coronavírus foi nomeado como SARS-CoV-2. que produz a doença classificada como COVID-19, sendo agente causador de uma série de casos de pneumonia em todos os cinco continentes, tornando-se uma pandemia mundial e a mesma, iniciou-se na cidade de Wuhan (China). O presente estudo teve como objetivo, fazer saber, sobre o conhecimento da epidemiologia e etiologia do Coronavírus (COVID-19), como ocorre a infecção, seus sinais, sintomas, diagnóstico e as recomendações para a prática do exercício e da atividade física. Essa pesquisa é do tipo documental. Seguir as orientações da Organização Mundial de Saúde (OMS) e dos demais órgãos e secretárias de saúde significa proteger-se e não ser infectado pelo Coronavírus e pode-se concluir, que o Governo Federal, através do Ministério da Saúde, vem agregar o profissional de Educação Física, em equipe multidisciplinar do Sistema Único de Saúde - SUS. Disposto sobre a Ação Estratégica "O Brasil Conta Comigo - Profissionais da Saúde", voltado para à capacitação e ao cadastramento de profissionais da área de saúde, sendo uma das áreas os professores de Educação Física do território nacional e estando os mesmos com o registro no Conselho Regional de Educação Física - CREF, para o enfrentamento à pandemia do Coronavírus (COVID-19), sendo de responsabilidade do Conselho Federal de Educação Física - CONFEF, confirmar o profissionais habilitados, junto ao Ministério da Saúde.

Palavras-chaves: Epidemiologia, COVID-19, Atividade Física.

\footnotetext{
${ }^{1}$ Professor de Educação Física SEM FRONTEIRAS DA FIEP-BRASIL / Delegado Adjunto Nacional da Federação Internacional de Educação Física FIEP / Secretario e Imortal da ABEF - Academia Brasileira de Educação Física / Conselheiro CREF 19AL / Professor de Educação Física do Instituto Federal de Alagoas/IFAL e doutorando em saúde coletiva com ênfase em Educação Física E-mail: cassiohartmann04@gmail.com

${ }^{2}$ Prof. Dr. Gabriel C. D. Lopes, PhD Professor e Orientador Doutor em Educação / PhD em Psicanálise Clínica Presidente da LUI - Logos University Int.Professor / Membro Imortal da ABEF - Academia Brasileira de Educação Física - E-mail: president@unilogos.education
} 
${ }^{3}$ Professor Co-orientador / Doutor em Ciências do Movimento Humano / Mestre em Educação Física / Especialista em Fisiologia do Exercício / Delegado Adjunto da Federação Internacional de Educação Física FIEP-PR. E-mail: vieira.fsf@gmail.com

\begin{abstract}
${ }^{4}$ Professor de Ciências e Médico / Bacharel em Medicina Poznan University of Medical Science, Poland/ Bacharel em Ciências Médicas e Laboratoriais (Cito-Tecnologia) University of Connecticut, Storrs, CT, USA / Especialista em Clínica Geral Queen Mary University / Especialista em Urgência e Emergência Medvarsity-Apollo Hospital / Doutorado em Liderança e Gestão Estratégica London School of Internation Business / Doutorado PhD: Pan-American University - Health Care Management / Doutorado PhD: Swiss Open University in Economics. E-mail: besson123@yahoo.com
\end{abstract}

\title{
ABSTRACT
}

The new Coronavirus has been named SARS-CoV-2. which produces the disease classified as COVID-19, being the causative agent of a series of pneumonia cases in all five continents, becoming a worldwide pandemic and the same, started in the city of Wuhan (China). This study aimed to make known, about the knowledge of the epidemiology and etiology of the Coronavirus (COVID-19), how the infection occurs, its signs, symptoms, diagnosis and the recommendations for the practice of exercise and physical activity. This research is of the documentary type similar to bibliographic research. Following the guidelines of the World Health Organization (WHO) and other health agencies and secretaries means protecting yourself and not being infected with the Coronavirus and it can be concluded that the Federal Government, through the Ministry of Health, comes to add the professional of Physical Education, in a multidisciplinary team of the Unified Health System - SUS. Where you can find out about the Strategic Action "Brazil Counts with Me - Health Professionals", aimed at training and registering health professionals, one of the areas being Physical Education teachers from the national territory and the same ones with the registration at the Regional Council of Physical Education - CREF, to face the Coronavirus pandemic (COVID-19), being the responsibility of the Federal Council of Physical Education - CONFEF, to confirm the qualified professionals, with the Ministry of Health.

Keywords: Epidemiology, COVID-19, Physical Activity.

\section{RESUMEN}

El nuevo Coronavirus ha sido nombrado SARS-CoV-2. que produce la enfermedad clasificada como COVID-19, siendo el agente causante de una serie de casos de neumonía en los cinco continentes, convirtiéndose en una pandemia mundial y lo mismo, comenzó en la ciudad de Wuhan (China). Este estudio tuvo como objetivo dar a conocer, sobre el conocimiento de la epidemiología y etiología del Coronavirus (COVID-19), cómo se produce la infección, sus signos, síntomas, diagnóstico y las recomendaciones para la práctica del ejercicio y la actividad física. Esta investigación es del tipo documental similar a la investigación bibliográfica. Seguir las pautas de la Organización Mundial de la Salud (OMS) y otras agencias y secretarias de salud significa protegerse y no estar infectado con el Coronavirus y se puede concluir que 
el Gobierno Federal, a través del Ministerio de Salud, viene a agregar al profesional de Educación Física, en un equipo multidisciplinario del Sistema Único de Salud SUS. Donde puede encontrar información sobre la Acción Estratégica "Brasil cuenta conmigo - Profesionales de la salud", dirigida a capacitar y registrar profesionales de la salud, una de las áreas son los maestros de Educación Física del territorio nacional y los mismos con el registro en el Consejo Regional de Educación Física - CREF, para enfrentar la pandemia de Coronavirus (COVID-19), siendo responsabilidad del Consejo Federal de Educación Física - CONFEF, confirmar a los profesionales calificados, con el Ministerio de Salud.

Palabras clave: Epidemiología, COVID-19, Actividad Física.

\section{INTRODUÇÃO}

Epidemiologia, ou a ciência das epidemias, propõe-se estudar quantitativamente a distribuição dos fenômenos de saúde/doença, e seus fatores condicionantes e determinantes, nas populações humanas.

A epidemiologia é um campo da ciência que trata dos vários fatores genéticos, sociais ou ambientais e condições derivados de exposição microbiológica, tóxica, traumática, etc. que determinam a ocorrência e a distribuição de saúde, doença, defeito, incapacidade e morte entre os grupos de indivíduos.

Portanto a epidemiologia tem relação direta e imediata com a área de Educação Física e segundo Hallal \& Knuth (2011) uma das principais limitações da área de epidemiologia da atividade física se refere à conceituação, visto que existe uma abundância de termos utilizados para classificar os indivíduos de determinado estudo em termos de sua prática de atividade física.

E com o a propagação da pandemia o Ministério da Saúde publicou no Diário Oficial da União a Portaria № 639, de 31 de março de 2020, dispondo sobre a Ação Estratégica "O Brasil Conta Comigo - Profissionais da Saúde", voltada à capacitação e ao cadastramento de profissionais da área de saúde para o enfrentamento à pandemia do Coronavírus (COVID-19). Dentre eles estão os Profissionais de Educação Física, visando auxiliar os gestores federais, estaduais, e municipais do Sistema Único de Saúde (SUS).

Este estudo tem como objetivo fazer saber, sobre o conhecimento da epidemiologia e etiologia do Coronavírus (COVID-19), como ocorre a infecção, seus sinais, sintomas, diagnóstico e as recomendações para a prática do exercício e da atividade física. 
Caracteriza-se como pesquisa qualitativa, segundo Marconi \& Lakatos (2017) aquela que identifica fatores relevantes de um objeto de maneira a analisar o teor de documentos ou realidades, e ainda ressaltam a importância do embasamento teórico, assim sendo, o atual artigo mostra uma realidade que vem acontecendo nos cinco continentes, acompanhado de revisão bibliográfica e por documentos inéditos referentes ao novo Coronavírus.

\section{CORONAVÍRUS (COVID-19)}

No fim de 2019, o novo Coronavírus foi nomeado como SARS-CoV-2. Este Novo Coronavírus produz a doença classificada como COVID-19, sendo agente causador de uma série de casos de pneumonia na cidade de Wuhan, na China (MCINTOSH \& NOVEL, 2020).

A Organização Mundial da Saúde (OMS) declarou, em 30 de janeiro de 2020, que o surto da doença causada pelo novo Coronavírus (COVID-19) constitui uma Emergência de Saúde Pública de Importância Internacional - o mais alto nível de alerta da Organização, conforme previsto no Regulamento Sanitário Internacional. Em 11 de março de 2020, a COVID-19 foi caracterizada pela OMS como uma pandemia. Segundo Mclntosh \& Novel (2020), ainda não há informações plenas sobre a história natural nem medidas de efetividade inquestionáveis para manejo clínico dos casos de infecção humana pelo SARS-CoV-2, restando ainda muitos detalhes a serem esclarecidos. No entanto, sabe-se que o vírus tem alta transmissibilidade e provoca uma síndrome respiratória aguda que varia de casos leves cerca de $80 \%$ - a casos muito graves com insuficiência respiratória - entre 5\% e 10\% dos casos. Sua letalidade varia, principalmente, conforme a faixa etária (Quadro 1) e condições clínicas associadas.

Quadro 1 - Letalidade provocada pela COVID-19 por faixa etária na China, Ministério da Saúde, 2020. 


\section{Taxa de letalidade por idade:}

- 0,2\% em pacientes entre 10 e 19 anos

- 0,2\% em pacientes entre 20 e 29 anos

- $0,2 \%$ em pacientes entre 30 e 39 anos

- 0,4\% em pacientes entre 40 e 49 anos

- $1,3 \%$ em paciente entre 50 e 59 anos

- 3,6\% em paciente entre 60 e 69 anos

- $8.0 \%$ em pacientes entre 70 e 79 anos

- $14.8 \%$ em pacientes acima ou igual a 80 anos

Ref: CDC China Weekly. Accessed Feb 20, 2020.

Portanto é necessário agir e, para esse fim, as melhores e mais recentes evidências foram utilizadas na redação deste documento. Pela dinâmica da epidemia e da produção de conhecimento associada a ela, as informações podem sofrer alterações conforme avance o conhecimento sobre a doença. Dessa forma, este protocolo específico para serviços de Atenção Primária à Saúde / Estratégia Saúde da Família (APS/ESF) nos cenários de transmissão comunitária vai ser atualizado sempre que necessário.

Considerando a existência de fase de transmissão comunitária da COVID-19, é imprescindível que os serviços de APS/ESF trabalhem com abordagem sindrômica do problema, não exigindo mais a identificação do fator etiológico por meio de exame específico.

\subsection{INFECÇÃO}

A infecção humana provocada pelo SARS-CoV-2 é uma zoonose. O vírus é classificado como um beta Coronavírus do mesmo subgênero da Síndrome Respiratória do Oriente Médio (MERS), porém de outro subtipo (MCINTOSH \& NOVEL, 2020). A transmissão do SARS-CoV-2 de humanos para humanos foi confirmada na China e nos EUA e ocorre principalmente com o contato de gotículas respiratórias oriundas de pacientes doentes e sintomáticos. A transmissão do vírus por indivíduos assintomáticos segue em controvérsia até o presente momento, mas, sabe-se que em média, o período de incubação do vírus é estimado em 5 a 6 dias, mas com variação aceitável de 0 a 14 dias (ROTHE, 2020). 


\subsection{SINAIS E SINTOMAS}

O paciente com a doença COVID-19 apresenta geralmente os seguintes sintomas e sinais (LEUNG et al., 2004; CHAN, et al., 2020):

- Febre $\left(>37,8^{\circ} \mathrm{C}\right)$;

- Tosse;

- Dispneia;

- Mialgia e fadiga;

- Sintomas respiratórios superiores; e

- Sintomas gastrointestinais, como diarreia (mais raros).

O quadro clínico, típico de uma Síndrome Gripal (SG), pode variar seus sintomas desde uma apresentação leve e assintomática (não se sabe a frequência), principalmente em jovens adultos e crianças, até uma apresentação grave, incluindo choque séptico e falência respiratória (CHAN, et al., 2020). A maior parte dos casos em que ocorreu óbito foi em pacientes com alguma comorbidade pré-existente $(10,5 \%$ doença cardiovascular, $7,3 \%$ diabetes, 6,3\% doença respiratória crônica, 6\% hipertensão e 5,6\% câncer e/ou idosos, conforme descrito no quadro 2 (WANG et al., 2020).

Quadro 2. Comorbidades que contraindicam acompanhamento ambulatorial da Síndrome Gripal em APS/ESF, Ministério da Saúde, 2020.

\section{COMORBIDADES QUE INDICAM AVALIAÇÃO EM CENTRO DE REFERÊNCIA}

Doenças cardíacas descompensadas

- Doença cardíaca congênita

- Insuficiência cardíaca mal controlada

- Doença cardíaca isquêmica descompensada

- Doenças respiratórias descompensadas

- DPOC e asma mal controlados

- Doenças pulmonares intersticiais com complicações

- Fibrose cística com infecções recorrentes

- Displasia broncopulmonar com complicações

- Crianças com doença pulmonar crônica da prematuridade

- Doenças renais crônicas em estágio avançado (graus 3, 4 e 5)

- Pacientes em diálise

- Imunossupressos

- Transplantados de órgãos sólidos e de medula óssea

- Imunossupressão por doenças e/ou medicamentos (em vigência de quimioterapia/radioterapia, entre outros medicamentos) 
- Portadores de doenças cromossômicas e com estados de fragilidade imunológica (ex.: Síndrome de Down)

- Diabetes (conforme juízo clínico)

- Gestante de alto risco

Huang et al., (2020) demonstra que a taxa de letalidade está em torno de 3,8\% na China, porém o valor varia conforme o país, e, epidemiologicamente, homens entre 41 e 58 anos representam a maioria dos casos de pacientes confirmados, sendo febre e tosse os sintomas mais presentes. As alterações em exames complementares mais comuns são infiltrados bilaterais nos exames de imagem de tórax, linfopenia no hemograma e aumento da proteína C-reativa. A doença apresenta fundamentalmente complicações respiratórias: pneumonia e Síndrome da Angústia Respiratória Aguda SARA.

\subsection{DIAGNÓSTICO}

As definições de caso e critérios clínicos para a avaliação diagnóstica ainda não são consenso entre os especialistas (MCINTOSH \& NOVEL, 2020), entretanto, pode-se avaliar o quadro da COVID-19 de maneira clínica e laboratorial.

O quadro clínico inicial da doença é caracterizado como Síndrome Gripal conforme sinais e sintomas evidenciados anteriormente. O diagnóstico sindrômico depende da investigação clínico-epidemiológica e do exame físico.

Conduta uniforme é sugerida para todos os casos de SG no contexto da APS/ESF, dada a impossibilidade de atestar com 100\% de segurança se a SG é causada pelo SARS-CoV-2 ou por outro vírus.

O diagnóstico laboratorial é realizado por meio das técnicas de transcriptasereversa Polymerase Chain Reaction (RT-PCR), em tempo real e sequenciamento parcial ou total do genoma viral. Na fase atual de mitigação da epidemia, nos cenários de transmissão comunitária, o diagnóstico etiológico só será realizado em casos de Síndrome Respiratória Aguda Grave, junto a serviços de urgência/emergência ou hospitalares.

A comorbidade patogênica ocorre quando duas ou mais doenças estão etiologicamente relacionadas; comorbidade diagnóstica ocorre quando as manifestações da doença associada forem similares às da doença primária; e 
comorbidade prognóstica ocorre quando houver doenças que predispõem o paciente a desenvolver outras doenças.

\section{RECOMENDAÇÕES DA PRÁTICA DE ATIVIDADE FíSICA E EXERCíCIO Físıco}

Atualmente vários profissionais de Educação Física que estão em cargos de gestão, seja na esfera Federal, Estadual, Municipal ou no respectivo conselho de classes Sistema CONFEF/CREFs (CONSELHO FEDERAL DE EDUCAÇÃO FíSICA E CONSELHO REGIONAL DE EDUCAÇÃO FíSICA) fazem a interlocução da atividade física com o Ministério da Saúde.

Desde a Política Nacional de Promoção da Saúde (BRASIL, 2006), editais e portarias anuais estimularam municípios e estados a promoverem estratégias populacionais em atividade física e outros temas, com abordagem integrada, intersetorial e de participação social. Nessas condições, foi formada uma Rede Nacional de Atividade Física (BECKER, GONÇALVES \& REIS, 2016), que além de estar atrelada aos serviços de saúde locais, dialogando com o Ministério da Saúde, recebe apoio e parceria do Centro de Controle e Prevenção de Doenças Norte Americano, que trabalha em várias frentes com o Ministério da Saúde no Brasil e Universidades parceiras.

Ter cuidado com a saúde mental e física é essencial para lutar contra o Coronavírus - COVID 19, que em 11 de março de 2020 a COVID-19 foi caracterizada pela OMS como uma pandemia, causando no mundo milhares de mortes. Esse é um dos principais conselhos de Tedros Adhanom, diretor geral da Organização Mundial da Saúde - OMS, e após mais de sessenta (60) dias dessa entrevista, em maio de 2020, esse número ultrapassa 297.119 de mortes mundialmente.

Em coletiva de imprensa em Genebra na Suíça, (TEDROS, 2020):

Alerta sobre os benefícios de ter uma vida saudável. "Nesse tempo difícil, é importante fazer exercícios físicos, no caso dos adultos por 30 minutos e das crianças 1 hora por dia", Caso não possa sair de casa, devido à quarentena, o diretor da Organização Mundial de Saúde (OMS) indica que as pessoas encontrem alternativas, como fazer ioga, com acompanhamento online, ou mesmo subir e descer as escadas. 
De acordo com Tedros, seguir as recomendações dos órgãos de saúde, como praticar os exercícios e atividades físicas em áreas ao ar livre, com pouca circulação de pessoas, ou mesmo em casa, ter cuidado com ambientes infectados são orientações de suma importância para não contaminação e não proliferação do vírus.

A atividade física regular, faz bem para o sistema imunológico e deve-se evitar exercícios muito fortes que levem a exaustão, podendo fazer com que baixe a imunidade, expondo o praticante a infecções como o COVID-19.

Para os indivíduos que são ativos é importante manter a rotina de treino sem alterar a intensidade, volume, frequência e o tempo. Já para os indivíduos sedentários é importante iniciar com exercícios leves e dê preferência com a orientação de um Professor de Educação Física.

Os exercícios ou atividades físicas devem ser realizados no mínimo três (03) vezes por semana e com distanciamento social de pelo menos $1,50 \mathrm{~cm}$ respeitando as orientações e recomendações da Organização Mundial de Saúde (OMS) e órgãos de saúde.

\section{CONSIDERAÇÕES FINAIS}

É notório o diferencial do Profissional de Educação Física, que primordialmente e na visão epidemiológica vem contribuir axiologicamente com as equipes multidisciplinares no Sistema Único de Saúde - SUS e fica evidente através da União/Governo Federal e da Portaria Ministerial № 639, de 31 de março de 2020, dispondo sobre a Ação Estratégica "O Brasil Conta Comigo - que o professor de Educação Física é de suma importância e relevante para o combate e enfrentamento à pandemia do Coronavírus (COVID-19), frente ao Sistema Único de Saúde (SUS).

Faz-se necessário, que o Professor de Educação Física, conheça o quadro de comorbidade, seja ela patogênica, diagnóstica ou prognóstica, para que saiba qual instrumento e procedimento utilizar em cada momento da prescrição da atividade e do exercício físico para com o paciente, de modo que o mesmo venha se recuperar progressivamente, seguindo as orientações da Organização Mundial de Saúde (OMS) e dos demais órgãos e secretárias de saúde, levando em consideração todas as vertentes de higienização para que o profissional da área de saúde, não venha se infectar com o Coronavírus (COVID-19). 
Diante das informações nesse artigo, sugere-se que pesquisas sejam realizadas com pacientes de COVID-19 para que se possa mostrar, seja, em pesquisa qualitativa ou quantitativa, quais os efeitos de um programa de atividades e/ou exercícios físicos com portadores que foram acometidos com o Coronavírus. 


\section{REFERÊNCIAS}

BECKER, L.A., GONÇALVES, P.B., REIS, R.S. Programas de promoção de atividade física no Sistema Único de Saúde brasileiro: revisão sistemática. Revista Brasileira de Atividade Física \& Saúde; 21(2):110-122, 2016.

BRASIL, MINISTÉRIO DA SAÚDE. LEI № 13.979, DE 6 DE FEVEREIRO DE 2020. http://www.in.gov.br/en/web/dou/-/lei-n-13.979-de-6-de-fevereiro-de-2020242078735. Acesso em: 11 de maio. 2020.

BRASIL. MINISTÉRIO DA SAÚDE. Política Nacional de Promoção da Saúde. Secretaria de Vigilância em Saúde. Ministério da Saúde, Secretaria de Atenção à Saúde. - Brasília: 2006.

CHAN, J.F., YUAN, S., KOK, K., TO, K.K., CHU, H., YANG, J. et al., A familial cluster of pneumonia associated with the 2019 novel coronavirus indicating personto- person transmission: a study of a family cluster. The Lancet Regional Health, Volume 395, p. 514-523, 2020.

GUAN, W., et al., Clinical Characteristics of Coronavirus Disease 2019 in China. The New England Journal Of Medicine, Vol. 382, n 13, 2020.

HALLAL, P. C., KNUTH, A.G. Epidemiologia Da Atividade Física E A Aproximação Necessária Com As Pesquisas Qualitativas. Rev. Bras. Ciênc. Esporte, Florianópolis, v. 33, n. 1, p. 181-192, jan./mar. 2011

HUANG, C., WANG, Y., LI, X., REN, L., ZHAO, J., HU, Y. et al. Clinical features of patients infected with 2019 novel coronavirus in Wuhan, China. The Lancet Regional Health, Volume 395, p. 497-506, 2020.

KUPFERSCHMIDT, K. Study claiming new coronavirus can be transmitted by people without symptoms was flawed. Science. February 3, 2020

LAKATOS, E.M. MARCONI, M.A. Fundamentos de Metodologia Científica. São Paulo: ebook, Atlas Editora, 2017.

LEUNG, G.M., HEDLEY, A.J., HO, L.M., et al., The epidemiology of severe acute respiratory syndrome in the 2003 Hong Kong epidemic: an analysis of all 1755 patients. Ann Intern Med, 141: 662-73, 2004.

LI, Q. et al., Early Transmission Dynamics In Wuhan, China, Of Novel CoronavirusInfected Pneumonia. The New England Journal Of Medicine, Vol. 382, n 13, 2020.

MCINTOSH, K. Coronavirus disease 2019 (COVID-19): Epidemiology, virology, clinical features, diagnosis and prevention. UpToDate, Inc, Wolters Kluwer, may 2020.

http://plataforma.saude.gov.br/novocoronavirus/ (referência para letalidade atual na china). 
RAZAI, M.S., DOERHOLT, K., LADHANI, S., OAKESHOTT, P. Coronavirus disease 2019 (COVID019): a guide for UK GPs. The BMJ, 2020.

ROTHE, C. et al., Letter To Editor: Transmission of 2019-nCoV Infection from an Asymptomatic Contact in Germany. The New England Journal Of Medicine, 2020.

SIMÕES, S.F. FERNANDES FILHO, J.. Análise dos estudos epidemiológicos em atividade física no nordeste brasileiro. FIEP Bulletin On-line, v. 86, n. 2, 2016.

WANG, C., HORBY, P.W., HAYDEN, F.G., GAO, G.F. A novel coronavirus outbreak of global health concern. The Lancet Regional Health, Volume 395, p. 470-473, 2020.

WHO - World Health Organization. Q \& A On Coronavirus, disponível em: https:/www.who.int/emergencies/diseases/novel-coronavirus-2019. Acesso em: 08 de maio. 2020. 